\title{
Performance Analysis of the Threshold Digital Relaying M2M System
}

\author{
Lingwei $\mathrm{Xu}^{1}$, Hao Zhang ${ }^{1,2}$ and T. Aaron Gulliver ${ }^{2}$ \\ ${ }^{1}$ College of Information Science and Engineering, Ocean University of China, \\ Qingdao 266100, China \\ ${ }^{2}$ Department of Electrical and Computer Engineering, University of Victoria, \\ Victoria V8W 3P6, Canada \\ gaomilaojia2009@163.com; zhanghao@ouc.edu.cn; agullive@ece.uvic.ca
}

\begin{abstract}
Exact closed-form outage probability $(O P)$ expressions are presented for multiplemobile relay-based mobile-to-mobile (M2M) systems with threshold digital relaying $(T D R)$ and relay selection over $N$-Nakagami fading channels. Numerical simulation is used to verify the accuracy of the analytic results. The effect of the fading coefficient, the number of cascaded components, the relative geometric gain, and the power-allocation on the $O P$ is examined.
\end{abstract}

Keywords: M2M communication, N-Nakagami fading channels, threshold digital relaying, outage probability, relay selection

\section{Introduction}

Mobile-to-mobile (M2M) communication has attracted wide research interest in recent years. It is widely employed in many popular wireless communication systems, such as mobile ad-hoc networks and vehicle-to-vehicle networks [1]. When both the transmitter and receiver are in motion, the double-Rayleigh fading model has been shown to be applicable [2]. Extending this model to the more realistic Nakagami fading, a doubleNakagami fading model has also been considered [3]. A generalization of this model, the $\mathrm{N}$-Nakagami distribution, which is the product of $N$ Nakagami random variables which are statistically independent but not necessarily identically distributed, is introduced and analyzed in [4].

Error propagation limits the end-to-end (E2E) performance of digital relaying. Error detection is an important way to mitigate error propagation, but it increases both the relay processing and delay. When error detection is either unavailable or inefficient, threshold digital relaying (TDR) can be employed using the instantaneous signal-to-noise ratio (SNR) of the source-relay link as an indication of the reliability of the signal at the relay. If the source-relay SNR is larger than the threshold, the probability of error at the relay is small and hence the relay retransmits the signal. Otherwise, the relay does nothing. In [5], the optimal threshold function that minimizes the E2E bit error rate (BER) was derived analytically. It was shown that if the threshold is selected properly, TDR can improve the BER performance significantly compared to simple relaying. In [6], the asymptotic E2E BER with TDR was derived relative to the optimal threshold over Rayleigh fading channels. Approximations for the optimal threshold that minimizes the E2E BER were derived in [7], and the resulting BER performance was derived analytically for BPSK modulation. These results show that using a TDR can significantly reduce the E2E BER compared to simple digital relaying.

Relay selection has emerged as a powerful technique for improving the performance of a wireless communication system without increasing the transmitted power. Relay selection has been studied extensively in the literature, see, e.g., [8-10] and the references 
therein. The outage probability (OP) performance of a relay selection scheme for decodeand-forward (DF) relaying in a cooperative vehicular network over double-Rayleigh fading channels was investigated in [11]. However, the direct transmission link between the source and destination was ignored. To the best knowledge of our knowledge, the OP of a multiple-mobile relay-based TDR system with relay selection over N-Nakagami fading channels has not been investigated. In [5-7], only a simple three node network with a source, destination and relay is considered. In this work, the analysis is presented for multiple relays. We also present the analysis for N-Nakagami fading channels which subsumes the double-Rayleigh fading considered in [11] as a special case. The direct transmission link between the source and destination is also considered. Exact closed form OP expressions are derived for threshold digital relaying over N-Nakagami fading channels.

The rest of the paper is organized as follows. The multiple-mobile relay-based M2M system model with relay selection is presented in Section 2. Section 3 provides the exact closed form OP expressions for threshold digital relaying. Section 4 presents Monte Carlo simulation results which verify the analysis in Section 3. Finally, some concluding remarks are given in Section 5.

\section{The System Model}

We consider a multi-mobile-node cooperation model, namely a single mobile source (MS) node, $L$ mobile relay (MR) nodes, and a single mobile destination (MD) node. The nodes operate in half-duplex mode, which are equipped with a single pair of transmitter and receiver antennas.

Following the approach in [5], we let $d_{\mathrm{SD}}, d_{\mathrm{SR} l}$, and $d_{\mathrm{RD} l}$ represent the distances of $\mathrm{MS} \rightarrow \mathrm{MD}, \mathrm{MS} \rightarrow \mathrm{MR}_{l}$, and $\mathrm{MR}_{l} \rightarrow \mathrm{MD}$ links, respectively. Assuming the path loss between $\mathrm{MS} \rightarrow \mathrm{MD}$ to be unity, the relative gain of $\mathrm{MS} \rightarrow \mathrm{MR}_{l}$ and $\mathrm{MR}_{l} \rightarrow \mathrm{MD}$ links are defined as $G_{\mathrm{SR} l}=\left(d_{\mathrm{SD}} / d_{\mathrm{SR} l}\right)^{v}$ and $G_{\mathrm{RD} l}=\left(d_{\mathrm{SD}} / d_{\mathrm{RD} l}\right)^{v}$, respectively, where $v$ is the path loss coefficient[12]. We further define the relative geometrical gain $\mu_{l}=G_{\mathrm{SR} l} / G_{\mathrm{RD} l}$ (in decibels), which indicates the location of the $l$ th relay with respect to the source and destination[5]. When the lth relay is close to the destination node, the values of $\mu_{l}$ are negative. When the $l$ th relay is close to the source node, the values of $\mu_{l}$ are positive. Also, when the $l$ th relay has the same distance to the source and destination nodes, $\mu_{l}$ is $0 \mathrm{~dB}$.

Let $h_{\mathrm{SD}}, h_{\mathrm{SR} l}$, and $h_{\mathrm{RD} l}$ represent the complex channel coefficients of $\mathrm{MS} \rightarrow \mathrm{MD}$, $\mathrm{MS} \rightarrow \mathrm{MR}_{l}$, and $\mathrm{MR}_{l} \rightarrow \mathrm{MD}$ links, respectively, which follow an N-Nakagami distribution. $h_{\mathrm{SD}}, h_{\mathrm{SR} l}$, and $h_{\mathrm{RD} l}$ are assumed to be a product of statistically independent, but not necessarily identically distributed, $N$ independent random variables

$$
h=\prod_{i=1}^{N} a_{i}
$$

where $N$ is the number of cascaded components, $a_{i}$ is a Nakagami distributed random variable with probability density function (PDF)

$$
f(a)=\frac{2 m^{m}}{\Omega^{m} \Gamma(m)} a^{2 m-1} \exp \left(-\frac{m}{\Omega} a^{2}\right)
$$

where $\Gamma(\cdot)$ is the Gamma function, $m$ is the fading coefficient and $\Omega$ is a scaling factor.

The PDF of $h$ is given by[4]

$$
f_{h}(h)=\frac{2}{h \prod_{i=1}^{N} \Gamma\left(m_{i}\right)} G_{0, N}^{N, 0}\left\lceil\left. h^{2} \prod_{i=1}^{N} \frac{m_{i}}{\Omega_{i}}\right|_{m_{1}, \ldots, m_{N}} ^{-}\right\rceil
$$

where $G[\cdot]$ is the Meijer's $G$-function .

Let $y_{\mathrm{SD}}=\left|h_{\mathrm{SD}}\right|^{2}, \quad y_{\mathrm{SR} l}=\left|h_{\mathrm{SR} l}\right|^{2}$, and $y_{\mathrm{RD} l}=\left|h_{\mathrm{RD} l}\right|^{2}$. The corresponding cumulative density functions (CDF) of $y$ can be derived as[4] 


$$
F_{y}(y)=\frac{1}{\prod_{i=1}^{N} \Gamma\left(m_{i}\right)} G_{1, N+1}^{N, 1}\left\lceil\left. y \prod_{i=1}^{N} \frac{m_{i}}{\Omega_{i}}\right|_{m_{1}, \ldots, m_{N}, 0} ^{1}\right\rfloor
$$

By taking the first derivative of (4) with respect to $y$, the corresponding PDF can be obtained as

$$
f_{y}(y)=\frac{1}{y \prod_{i=1}^{N} \Gamma\left(m_{i}\right)} G_{0, N}^{N, 0}\left\lceil\left. y \prod_{i=1}^{N} \frac{m_{i}}{\Omega_{i}}\right|_{m_{1}, \ldots, m_{N}} ^{-}\right\rfloor
$$

Based on the TDR cooperation protocol, the received signals $r_{\mathrm{SD}}$ and $r_{\mathrm{SR} l}$ at the MD and $\mathrm{MR}_{l}$ during the first time slot can be written as[6]

$$
\begin{gathered}
r_{S D}=\sqrt{K E} h_{S D} x+n_{D} \\
r_{S R l}=\sqrt{G_{S R l} K E} h_{S R l} x+n_{S R l}
\end{gathered}
$$

where $x$ denotes the transmitted signal, $n_{S R l}$ and $n_{\mathrm{D}}$ are the zero-mean complex Gaussian random variables with variance $N_{0} / 2$ per dimension. Here, $E$ is the total energy which is used by both source and relay terminals during two time slots. $K$ is the power-allocation parameter that controls the fraction of power reserved for the broadcasting phase. If $K=0.5$, the equal power allocation (EPA) scheme is used.

During the second time slot, only the best relay is activated in each transmission session. The best relay either retransmits the data or remains silent. In our scheme, the best relay is selected based on the following criterion

$$
\gamma_{R}=\max _{1 \leq l \leq L}\left(\gamma_{S R l}\right)
$$

where

$$
\gamma_{S R l}=\frac{K G_{S R l}\left|h_{S R l}\right|^{2} E}{N_{0}}=K G_{S R l}\left|h_{S R l}\right|^{2} \bar{\gamma}
$$

The best relay decides whether to decode and retransmit the signal by comparing the instantaneous SNR $\gamma_{R}$ to a threshold $\gamma_{T}$.

If $\gamma_{R}<\gamma_{T}$, the best relay remains silent. The output SNR at the destination can then be calculated as

where

$$
\gamma_{S C}=\gamma_{S D}
$$

$$
\gamma_{S D}=\frac{K\left|h_{S D}\right|^{2} E}{N_{0}}=K\left|h_{S D}\right|^{2} \bar{\gamma}
$$

If $\gamma_{R}>\gamma_{T}$, the best relay decodes the received signal and generates a signal $x_{1}$ and forwards $x_{1}$ to the MD. Based on DF cooperation protocol, the received signal at the MD is therefore given by

$$
r_{R D}=\sqrt{(1-K) G_{R D} E} h_{R D} x_{1}+n_{R D}
$$

where $n_{\mathrm{RD}}$ is a conditionally zero-mean complex Gaussian random variable with variance $N_{0} / 2$ per dimension.

If selection combining (SC) method is used at the MD, the output SNR at the destination can then be calculated as [13]

where

$$
\gamma_{S C C}=\max \left(\gamma_{S D}, \gamma_{R D}\right)
$$

$$
\gamma_{R D}=\frac{(1-K) G_{R D}\left|h_{R D}\right|^{2} E}{N_{0}}=(1-K) G_{R D}\left|h_{R D}\right|^{2} \bar{\gamma}
$$


Thus, we can obtain the cumulative distribution function (CDF) of the output SNR at the destination as

$$
F(r)=\operatorname{Pr}\left(\gamma_{R}<\gamma_{T}\right) \operatorname{Pr}\left(\gamma_{S C}<r \mid \gamma_{R}<\gamma_{T}\right)+\operatorname{Pr}\left(\gamma_{R}>\gamma_{T}\right) \operatorname{Pr}\left(\gamma_{S C C}<r \mid \gamma_{R}>\gamma_{T}\right)
$$

As $\gamma_{R}, \gamma_{S D}$ and $\gamma_{R D}$ are mutually independent random variables, (15) can be simplified and derived as follows:

$$
\begin{aligned}
F(r) & =\operatorname{Pr}\left(\gamma_{R}<\gamma_{T}\right) \operatorname{Pr}\left(\gamma_{S C}<r\right)+\operatorname{Pr}\left(\gamma_{R}>\gamma_{T}\right) \operatorname{Pr}\left(\gamma_{S C C}<r\right) \\
& =F_{\gamma_{R}}\left(\gamma_{T}\right) F_{\gamma_{S D}}(r)+\left(1-F_{\gamma_{R}}\left(\gamma_{T}\right)\right) F_{\gamma_{S C C}}(r)
\end{aligned}
$$

\section{The OP for M2M System}

In this section, the exact closed form OP expressions for the multiple-mobile relaybased M2M system with relay selection are derived.

The CDF of the $\gamma_{\mathrm{SD}}$ can be given as[4]

$$
F_{\gamma_{S D}}(r)=\frac{1}{\prod_{i=1}^{N} \Gamma\left(m_{i}\right)} G_{1, N+1}^{N, 1}\left\lceil\left.\frac{r}{\overline{\gamma_{S D}}} \prod_{i=1}^{N} \frac{m_{i}}{\Omega_{i}}\right|_{m_{1}, \ldots, m_{N}, 0} ^{1}\right\rfloor
$$

where

$$
\overline{\gamma_{S D}}=K \bar{\gamma}
$$

The CDF of the $\gamma_{\mathrm{R}}$ can be given as

$$
\begin{aligned}
& F_{\gamma_{R}}(r)=\prod_{l=1}^{L} P\left(\gamma_{S R l} \leq r\right)=\prod_{l=1}^{L} F_{\gamma_{S R l}}(r) \\
& =\prod_{l=1}^{L} \frac{1}{\prod_{j=1}^{N} \Gamma\left(m_{j}\right)} G_{1, N+1}^{N, 1}\left\lfloor\left.\frac{r}{\overline{\gamma_{S R l}}} \prod_{j=1}^{N} \frac{m_{j}}{\Omega_{j}}\right|_{m_{1}, \ldots, m_{N}, 0} ^{1}\right\rfloor
\end{aligned}
$$

where

$$
\overline{\gamma_{S R l}}=K G_{S R l} \bar{\gamma}
$$

The CDF of the $\gamma_{\mathrm{SCC}}$ can be given as

$$
\begin{aligned}
& F_{\gamma_{S C C}}(r)=P\left(\max \left(\gamma_{S D}, \gamma_{R D}\right) \leq r\right) \\
&= P\left(\gamma_{S D} \leq r\right) P\left(\gamma_{R D} \leq r\right) \\
&= \frac{1}{N} G_{1, N+1}^{N, 1}\left\lceil\left.\frac{r}{\overline{\gamma_{S D}}} \prod_{i=1}^{N} \frac{m_{i}}{\Omega_{i}}\right|_{m_{1}, \ldots, m_{N}, 0} ^{1}\right\rfloor \\
& \times \frac{1}{\prod_{i=1}^{N} \Gamma\left(m_{i}\right)} G_{1, N+1}^{N, 1}\left\lceil\left.\frac{r}{\overline{\gamma_{R D}}} \prod_{i=1}^{N} \frac{m_{j j}}{\Omega_{j j}}\right|_{m_{1}, \ldots ., m_{N}, 0} ^{1}\right\rfloor \\
& \prod_{j j=1} \Gamma\left(m_{j j}\right)
\end{aligned}
$$

where

$$
\overline{\gamma_{R D}}=(1-K) G_{R D} \bar{\gamma}
$$

The OP of the multiple-mobile relay-based M2M system can be expressed as 


$$
\begin{aligned}
& F\left(\gamma_{t h}\right)=\frac{1}{\prod_{i=1}^{N} \Gamma\left(m_{i}\right)} G_{1, N+1}^{N, 1}\left\lceil\left.\frac{\gamma_{t h}}{=} \prod_{\gamma_{S D}}^{N} \frac{m_{i}}{\Omega_{i}}\right|_{m_{1}, \ldots, m_{N}, 0} ^{1}\right\rfloor \times
\end{aligned}
$$

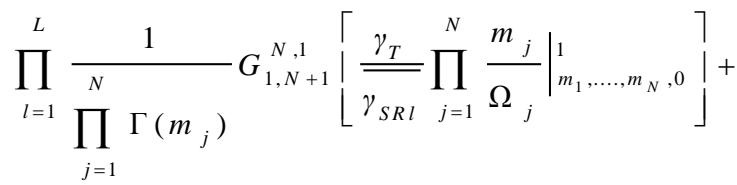

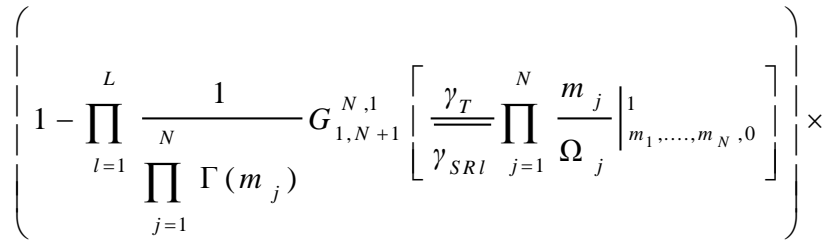

$$
\begin{aligned}
& \frac{1}{\prod_{i=1}^{N} \Gamma\left(m_{i}\right)} G_{1, N+1}^{N, 1}\left\lceil\left.\frac{\gamma_{t h}}{=} \prod_{\frac{\gamma_{S D}}{N}} \frac{m_{i}}{\Omega_{i}}\right|_{m_{1}, \ldots, m_{N}, 0} ^{1}\right\rfloor \times \\
& \frac{1}{\prod_{j j=1}^{N} \Gamma\left(m_{j j}\right)} G_{1, N+1}^{N, 1}\left\lceil\frac{\gamma_{t h}}{\underline{\underline{\gamma_{R D}}}} \prod_{i=1}^{N} \frac{m_{j j}}{\Omega_{j j}}||_{m_{1}, \ldots, m_{N}, 0}\right\rfloor
\end{aligned}
$$

where $\gamma_{\text {th }}$ is a given threshold.

\section{Numerical Results}

In this section, some numerical results are presented to illustrate and verify the OP results obtained in the previous sections.

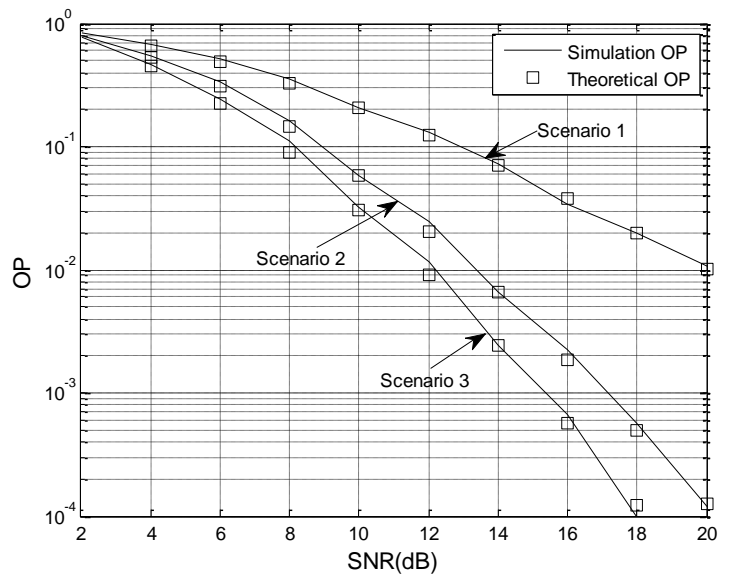

Figure 1. The OP Performance over N-Nakagami Fading Channels

Figure 1 presents the OP performance of the multiple-mobile relay-based M2M system over N-Nakagami fading channels. The relative geometrical gain $\mu=0 \mathrm{~dB}$. The powerallocation parameter $K=0.5$. The number of cascaded components $N=2$. The number of mobile relays $L=2$. The given threshold $\gamma_{\mathrm{th}}=4 \mathrm{~dB}, \gamma_{\mathrm{T}}=4 \mathrm{~dB}$. Here, we consider the following scenarios based on the combinations of the number of cascaded components $N$ and fading coefficient $m$ :

(1) Scenario 1: $m_{\mathrm{SD}}=1, m_{\mathrm{SR} l}=1, m_{\mathrm{RD} l}=1$ and $N_{\mathrm{SD}}=2, N_{\mathrm{SR} l}=N_{\mathrm{RD} l}=2 .(l=1,2)$ 
(2)Scenario 2: $m_{\mathrm{SD}}=2, m_{\mathrm{SR} l}=2, m_{\mathrm{RD} l}=2$ and $N_{\mathrm{SD}}=2, N_{\mathrm{SR} l}=N_{\mathrm{RD} l}=2 .(l=1,2)$

(3) Scenario 3: $m_{\mathrm{SD}}=2, m_{\mathrm{SR} l}=3, m_{\mathrm{RD} l}=3$ and $N_{\mathrm{SD}}=3, N_{\mathrm{SR} l}=N_{\mathrm{RD} l}=2$. $(l=1,2)$

From Figure 1,we can obtain that, the numerical simulation results coincide with the theoretical results well, and the accuracy of the analytical results is verified. With the SNR increased, the OP performance is improved. For example, in scenario 2, when $\mathrm{SNR}=10 \mathrm{~dB}$, the OP is $6 \times 10^{-2}, \mathrm{SNR}=12 \mathrm{~dB}$, the $\mathrm{OP}$ is $2 \times 10^{-2}$.

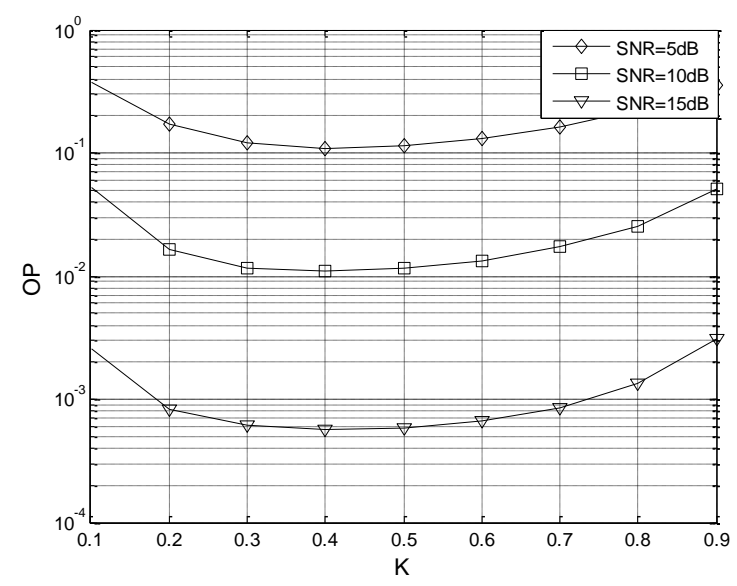

\section{Figure 2. The Effect of the Power-allocation Parameter $K$ on the OP Performance}

Figure 2 presents the effect of the power-allocation parameter $K$ on the OP performance of the multiple-mobile relay-based M2M system over N-Nakagami fading channels with various values of SNR. The number of cascaded components $N=2$. The fading coefficient $m=2$. The relative geometrical gain $\mu=0 \mathrm{~dB}$. The given threshold $\gamma_{\mathrm{th}}=4 \mathrm{~dB}, \gamma_{\mathrm{T}}=4 \mathrm{~dB}$. The number of mobile relays $L=2$.Simulation results show that the OP performance is improved with the SNR increased. For example, when $K=0.6, \mathrm{SNR}=5 \mathrm{~dB}$, the OP is $1.2 \times 10^{-1}, \mathrm{SNR}=10 \mathrm{~dB}$, the OP is $1.2 \times 10^{-2}, \mathrm{SNR}=15 \mathrm{~dB}$, the OP is $7 \times 10^{-4}$. When $\mathrm{SNR}=5 \mathrm{~dB}$, the optimum value of $K$ is 0.4 approximately; $\mathrm{SNR}=10 \mathrm{~dB}$, the optimum value of $K$ is 0.4 approximately; $\mathrm{SNR}=15 \mathrm{~dB}$, the optimum value of $K$ is 0.4 approximately.

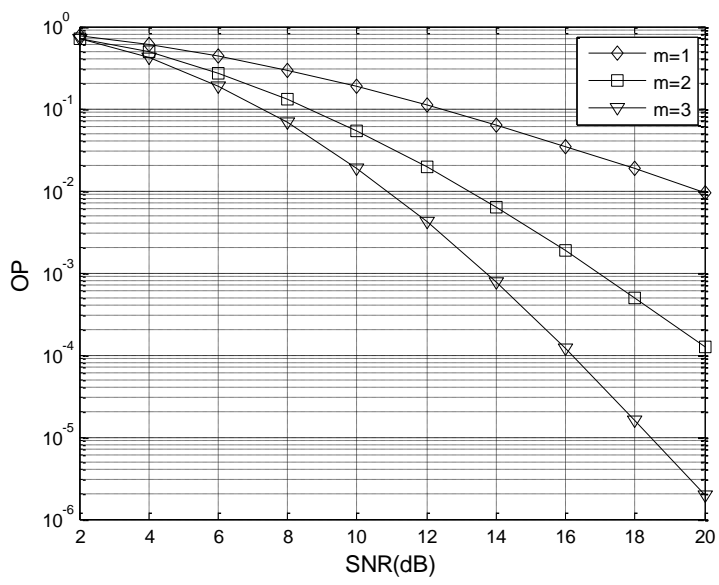

Figure 3. The Effect of the Fading Coefficient $\boldsymbol{m}$ on the OP Performance

Figure 3 presents the effect of the fading coefficient $m$ on the OP performance of the multiple-mobile relay-based M2M system over N-Nakagami fading channels. The number of cascaded components $N=2$.The fading coefficient $m=1,2,3$. The relative geometrical gain $\mu=0 \mathrm{~dB}$. The given threshold $\gamma_{\mathrm{th}}=4 \mathrm{~dB}, \gamma_{\mathrm{T}}=4 \mathrm{~dB}$. The power-allocation 
parameter $K=0.5$. The number of mobile relays $L=3$. Simulation results show that the OP performance is improved with the fading coefficient $m$ increased. For example, when $\mathrm{SNR}=12 \mathrm{~dB}, m=1$, the OP is $1 \times 10^{-1}, m=2$, the OP is $2 \times 10^{-2}, m=3$, the OP is $4 \times 10^{-3}$. When the $m$ is fixed, with the increase of SNR, the OP is reduced gradually.

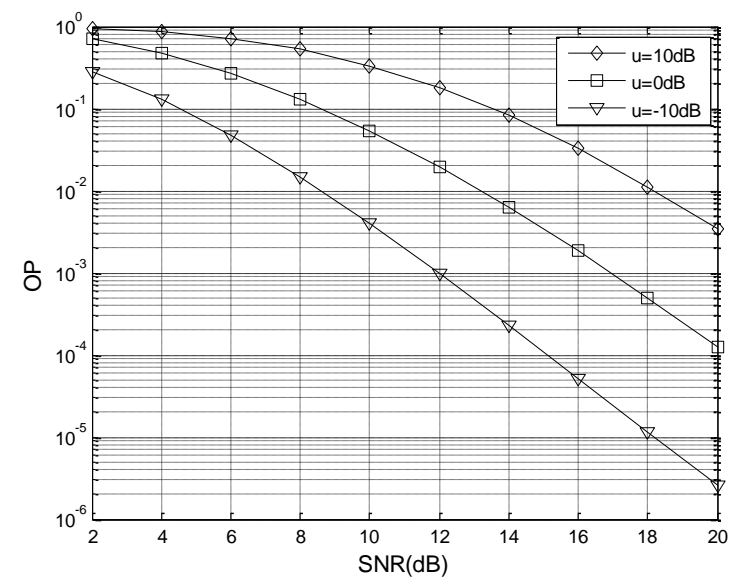

\section{Figure 4. The Effect of the Relative Geometrical Gain $\mu$ on the OP Performance}

Figure 4 presents the effect of the relative geometrical gain $\mu$ on the OP performance of the multiple-mobile relay-based M2M system over N-Nakagami fading channels. The number of cascaded components $N=2$. The fading coefficient $m=2$. The relative geometrical gain $\mu=10 \mathrm{~dB}, 0 \mathrm{~dB},-10 \mathrm{~dB}$. The given threshold $\gamma_{\mathrm{th}}=4 \mathrm{~dB}, \gamma_{\mathrm{T}}=2 \mathrm{~dB}$. The powerallocation parameter $K=0.5$. The number of mobile relays $L=2$.Simulation results show that the OP performance is improved as $\mu$ reduced. For example, when $\mathrm{SNR}=12 \mathrm{~dB}$, $\mu=10 \mathrm{~dB}$, the OP is $2 \times 10^{-1}, \mu=0 \mathrm{~dB}$, the OP is $2 \times 10^{-2}, \mu=-10 \mathrm{~dB}$, the OP is $1 \times 10^{-3}$. With the increase of SNR, the OP between them is reduced gradually.

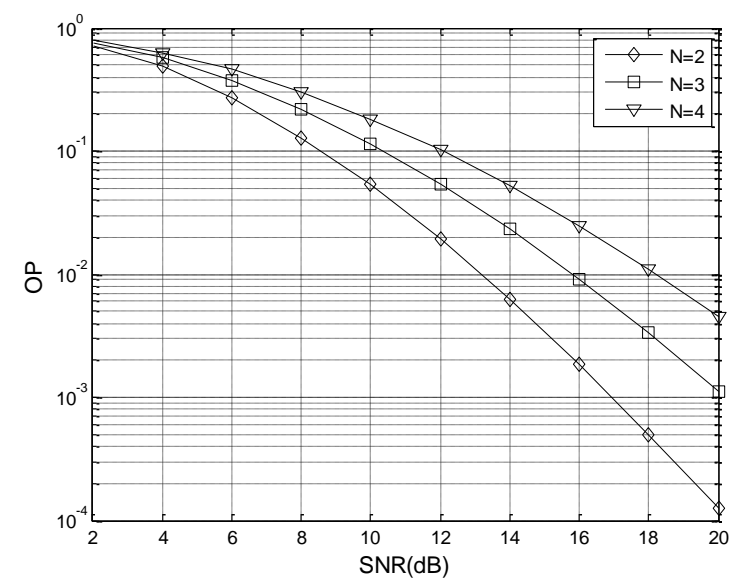

\section{Figure 5. The Effect of the Number of Cascaded Components $\mathbf{N}$ on the OP Performance}

Figure 5 presents the effect of the number of cascaded components $N$ on the OP performance of the multiple-mobile relay-based M2M system over N-Nakagami fading channels. The number of cascaded components $N=2,3,4$, which respectively denotes the 2-Nakagami, 3-Nakagami, 4-Nakagami fading channels. The fading coefficient $m=2$. The relative geometrical gain $\mu=0 \mathrm{~dB}$. The given threshold $\gamma_{\mathrm{th}}=4 \mathrm{~dB}, \gamma_{\mathrm{T}}=2 \mathrm{~dB}$. The powerallocation parameter $K=0.5$. The number of mobile relays $L=3$.Simulation results show 
that the OP performance is degraded as $N$ increased. For example, when $\mathrm{SNR}=12 \mathrm{~dB}$, $N=2$, the OP is $2 \times 10^{-2}, N=3$, the OP is $5.5 \times 10^{-2}, N=4$, the OP is $1 \times 10^{-1}$. This because the fading severity of the cascaded channels increases as $N$ increased. When the $N$ is fixed, with the increase of SNR, the OP is reduced gradually.

\section{Conclusions}

The exact closed-form OP expressions of the multiple-mobile relay-based M2M system over N-Nakagami fading channels are derived in this paper. The simulation results show that: the fading coefficient $m$, the number of cascaded components $N$, the relative geometrical gain $\mu$, and the power-allocation parameter $K$ have an important influence on the OP performance. The expressions derived here are simple to compute and thus complete and accurate performance results can easily be obtained with negligible computational effort. In the future, we will consider the impact of the correlated channels on the OP performance of the multiple-mobile relay-based M2M system.

\section{Acknowledgements}

The authors would like to thank the referees and editors for providing very helpful comments and suggestions. This project was supported by National Natural Science Foundation of China (no. 61304222, no. 60902005), Natural Science Foundation of Shandong Province (no.ZR2012FQ021), Shandong Province Higher Educational Science and Technology Program (no.J12LN88), International Science \& Technology Cooperation Program of Qingdao (no.12-1-4-137-hz).

\section{References}

[1] G. Wu, S. Talwar, K. Johnsson, N. Himayat and K. D. Johnson, "M2M: From mobile to embedded internet", IEEE Communications Magazine, vol. 49, no. 4, (2011).

[2] M. Uysal, "Diversity Analysis of Space-time Coding in Cascaded Rayleigh Fading Channels", IEEE Communication Letter, vol. 10, no. 3, (2006).

[3] F. K. Gong, J. Ge, and N. Zhang, "SER Analysis of the Mobile-Relay-Based M2M Communication over Double Nakagami-m Fading Channels", IEEE Communications Letters, vol. 15, no. 1, (2011).

[4] G. K. Karagiannidis, N. C. Sagias, and P. T. Mathiopoulos. N*Nakagami: a novel stochastic model for cascaded fading channels. IEEE Transactions on Communication, vol. 55, no. 8, (2007).

[5] H. Ilhan, M. Uysal, and I. Altunbas, "Cooperative Diversity for Intervehicular Communication: Performance Analysis and Optimization. IEEE Transactions on Vehicular Technology, vol. 58, no. 7, (2009).

[6] F. A. Onat, Y. J. Fan, H. Yanikomeroglu, and J. S. Thompson, "Asymptotic BER Analysis of Threshold Digital Relaying Schemes in Cooperative Wireless Systems", IEEE Transactions on Wireless Communications, vol. 7, no. 12, (2008).

[7] F. A. Onat, A. Adinoyi, Y. J. Fan, H. Yanikomeroglu, J. S. Thompson, and I. D. Marsland, "Threshold Selection for SNR-based Selective Digital Relaying in Cooperative Wireless Networks", IEEE Transactions on Wireless Communication, vol. 7, no. 11, (2008).

[8] D. S. Michalopoulos, and G. K. Karagiannidis, "Performance analysis of single-relay selection in rayleigh fading", IEEE Transactions on Wireless Communication, vol. 7, no. 10, (2008).

[9] S. Ikki and M. H. Ahmed, "Exact error probability and channel capacity of the best-relay cooperativediversity networks", IEEE Signal Processing Letters, vol. 16, no. 12, (2009).

[10] S. Ikki and M. H. Ahmed, "Performance analysis of adaptive decode-and-forward cooperative diversity networks with the best relay selection", IEEE Transactions on Communication, vol. 58, no. 1, (2010).

[11] M. Seyfi, S. Muhaidat, J. Liang, and M. Uysal, "Relay Selection in Dual-Hop Vehicular Networks", IEEE Signal Processing Letters, vol. 18, no. 2, (2011).

[12] W. F. Su, A. K. Sadek, and K. J. R. Liu, "Cooperative Communication Protocols in Wireless Networks: Performance Analysis and Optimum Power Allocation”, Wireless Personal Communications, vol. 44, no. 2, (2008).

[13] A. Papoulis, "Probability, Random Variables, and Stochastic Processes", McGraw-Hill, New York (1991). 


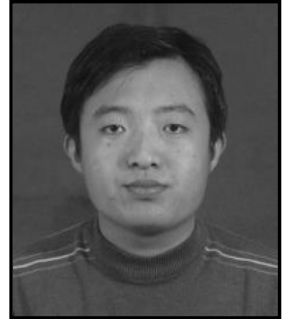

Lingwei Xu, he was born in Shandong, China, in 1987. He received his Bachelor Degree in Department of Communication and Electronics, Qingdao Technological University, China, in 2011.From 2011 to now, he is a student of College of Information Science and Engineering, Ocean University of China. His research interests include ultra-wideband radio systems, MIMO wireless systems, and $60 \mathrm{GHz}$ wireless communications.

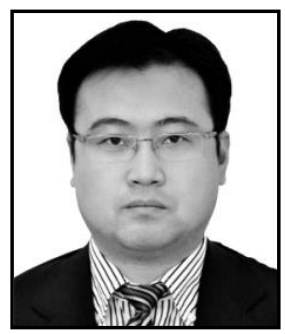

Hao Zhang, he was born in Jiangsu, China, in 1975. He received his Bachelor Degree in Telecom Engineering and Industrial Management from Shanghai Jiaotong University, China in 1994, his MBA from New York Institute of Technology, USA in 2001, and his $\mathrm{Ph} . \mathrm{D}$. in Electrical and Computer Engineering from the University of Victoria, Canada in 2004. His research interests include ultrawideband radio systems, MIMO wireless systems. He is a Professor in College of Information Science and Engineering, Ocean University of China.

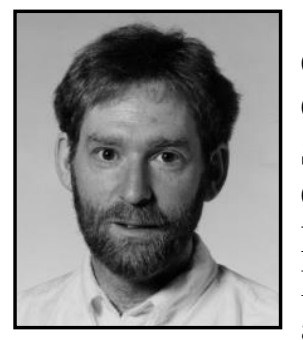

T. Aaron Gulliver, he received the Ph.D. degree in Electrical and Computer Engineering from the University of Victoria, Victoria, BC, Canada in 1989. From 1989 to 1991 he was employed as a Defense Scientist at Defense Research Establishment Ottawa, Ottawa, ON, Canada. He joined the University of Victoria in 1999 and is a Professor in the Department of Electrical and Computer Engineering. In 2002 he became a Fellow of the Engineering Institute of Canada, and in 2012 a Fellow of the Canadian Academy of Engineering. His research interests include information theory and communication theory. 
International Journal of Signal Processing, Image Processing and Pattern Recognition Vol.8, No.3 (2015) 